teil liegt in den notwendigen nachjustierenden Folge-Operationen während des Wachstums.

Die Hauptindikation für eine OP im Erwachsenenalter sind Schmerzen. Jedoch haben Operationen bei Erwachsenen eine erheblich höhere Komplikationsrate. Rückenmarkverletzungen sind in der Regel behandlungsbedingt. Im Erwachsenenalter kann Nervenwurzelkom- pression im Lumbalbereich durch degenerative Veränderungen auftreten: „Enge“ bei Bandscheiben- und FacettengelenkDegeneration.

Kommentar: Trotz aller Bemühungen ist die Ursache der AIS ungeklärt. Sie betrifft vorwiegend Mädchen und tritt familiär gehäuft auf. Sie sollte möglichst im präpubertären Alter erkannt werden, um konservativ behandeln zu können. Der entscheidende Zeitraum ist leider nicht durch eine gesetzlich verankerte Vorsorgeuntersuchung abgedeckt. Daher wird die Erkrankung oft erst spät aufgedeckt, was für die Betroffenen fatal ist!

Hannelore Willenborg

Supplement to Journal of Pediatric Orthopaedics 2011; 31 (1 Suppl)

\section{ALL: Welches Regime nach Rückfall?}

Für Kinder mit akuter lymphoblastischer Leukämie (ALL) ist die Prognose bei einem Rückfall immer noch relativ schlecht. Europäische Forscher haben in einer Multicenterstudie versucht, die Therapie zu optimieren.

$\mathrm{D}$ e Behandlung von Kindern mit rezidivierter ALL nach den Protokollen der Studiengruppe ALL-Relapse-Berlin/Frankfurt/Münster (ALL-REZ BFM) ist in Deutschland, Österreich und der Schweiz Standard. Dabei erhalten die Patienten alternierend kurze Polychemotherapiekurse (Block R1, R2) sowie kraniale/ kraniospinale Bestrahlung mit anschließender Erhaltungstherapie. In ALL-REZ BFM 90 ergänzte die Gruppe eine Chemotherapie mit hoch dosiertem Cytarabin und Etoposid im Block R3. 525 Kinder wurden in die Risikogruppen A (frühes Knochenmark-Rezidiv), B (spätes Knochenmark-Rezidiv) und C (isoliertes extramedulläres Rezidiv) eingeteilt und mit dem erweiterten Regime behandelt. Eine Gruppe von Patienten mit schlechter Prognose (PPG) konnte experimentell therapiert werden. 117 Kinder bekamen eine Stammzelltransplantation.

Die Wahrscheinlichkeit für ein ereignisfreies Überleben (pEFS) nach 10 Jahren betrug 0,30, für ein Gesamtüberleben 0,36 . Zwischen den drei Risikogruppen bestanden deutliche Unterschiede, so lag die pEFS in Gruppe A bei 0,17, in Gruppe $B$ bei 0,43 und in Gruppe $C$ bei 0,54, in PPG betrug die pEFS 0,15. Patienten der Hochrisikogruppen A plus PPG hatten einen höheren Nutzen von einer Stammzelltransplantation als von einer Chemotherapie (pEFS 0,33 vs. 0,$20 ; p=0,005$ ).

Die pEFS war ähnlich wie in den Studien ALL-REZ BFM 85/87. In multivariaten Analysen erwiesen sich Zeitpunkt und Lokalisation des Rückfalls, Immun- phänotyp sowie eine Stammzelltransplantation als signifikante Prädiktoren.

Kommentar: Die Entwicklung der Heilungschancen für Kinder mit Krebserkrankungen gehört zu den größten Erfolgsgeschichten in der Medizin der letzten Jahrzehnte. Etwa $70 \%$ aller unserer Patienten können heute dauerhaft geheilt werden. Die ALL ist die häufigste maligne Erkrankung im Kindesalter. Selbst nach einem Rückfall kann noch ein Drittel dieser Patienten erfolgreich behandelt werden. Die hier vorgestellten Therapiestudien gehören weltweit zu den größten und erfolgreichsten für Rückfälle bei ALL. Das wesentliche Prinzip hinter allen Verbesserungen der Überlebenschancen für Kinder mit onkologischen Erkrankungen war bisher eine stetige Intensivierung der Chemotherapie bei gleichzeitiger Verbesserung der Behandlung von Therapiekomplikationen.

Die hier vorgestellten Ergebnisse sind beispielhaft für viele Erkrankungen in der pädiatrischen Onkologie, bei denen diese Entwicklung an einem Endpunkt angelangt ist. Das Prinzip „Viel hilft viel“ lässt sich nicht mehr fortsetzen. Zwar konnten in bestimmten Patientengruppen die Ergebnisse durch eine Knochenmarktransplantation noch einmal verbessert werden, ein zusätzlicher intensiver Chemotherapie-Block führte aber nicht zu einer höheren Heilungschance.

Um die Behandlung krebskranker Kinder weiter zu verbessern, sind dringend neue Therapieansätze erforderlich, die die klassische Chemotherapie ergänzen und vielleicht sogar ablösen. Während Zytostatika mehr oder weniger alle Zellen angreifen, die sich in Teilung befinden, sollen neue Medikamente gezielt in die krankhafte Veränderung der Krebszellen eingreifen und somit mehr Wirksamkeit bei weniger Nebenwirkung erreichen. Aus der Grundlagenforschung gibt es zahlreiche Entwicklungen für solche Medikamente. Der Schritt in die klinische Anwendung ist sehr schwierig. Da die Veränderungen in den Krebszellen verschiedener Patienten sehr unterschiedlich sind, wird jedes neue Medikament nur für eine kleine Patientengruppe wirksam sein. Nur in internationaler Zusammenarbeit kann daher eine ausreichende Anzahl von Patienten behandelt werden, um die Wirksamkeit zu überprüfen.

Ein zweites Hindernis ist die zunehmende Bürokratisierung. Eine Wiederholung der Erfolgsgeschichte der letzten Jahrzehnte ist schon allein aufgrund des aktuellen Arzneimittelgesetztes nicht mehr möglich. Studien wie die hier publizierten, aus denen unser gesamtes Wissen über die Behandlung von Krebserkrankungen bei Kindern stammt, sind jetzt mit einem so astronomischen bürokratischen und damit auch finanziellen Aufwand verbunden, dass sie nur noch in wenigen Fällen durchführbar sind.

Neue Ideen sind also gefragt. Nicht nur bei Ärzten und Wissenschaftlern, sondern auch bei Politikern, die begangene Fehler zügig korrigieren sollten.

Prof. Dr. Daniel Steinbach/SUH

Tallen G et al. Long-term outcome in children with relapsed acute lymphoblastic leukemia after time-point and site-of-relapse stratification and intensified short-course multidrug chemotherapy: Results of trial ALL-REZ BFM 90. J Clin Oncol 2010; 28: 2339-47 